\title{
ANÁLISIS DE LA RELACIÓN ENTRE LA CONDUCTIVIDAD HIDRÁULICA EFECTIVA Y LA CURVA NÚMERO BAJO DOS INTENSIDADES DE LLUVIA
}

\author{
María Darder $^{(1)}$, Mario Castiglioni ${ }^{(2)(*)}$, Adrián Andriulo ${ }^{(1)}$ y Maria Sasal ${ }^{(3)}$ \\ ${ }^{(1)}$ Estación Experimental Agropecuaria Pergamino, Instituto Nacional de Tecnología Agropecuaria (INTA). \\ Pergamino, Buenos Aires, Argentina. \\ ${ }^{(2)}$ Cátedra de Manejo y Conservación de Suelos, Facultad de Agronomía, Universidad de Buenos Aires. \\ Buenos Aires, Buenos Aires, Argentina. \\ ${ }^{(3)}$ Estación Experimental Agropecuaria Paraná, Instituto Nacional de Tecnología Agropecuaria (INTA). \\ Paraná, Entre Ríos, Argentina. \\ ${ }^{(*)}$ e-mail: castigli@agro.uba.ar
}

\begin{abstract}
RESUMEN
La conductividad hidráulica efectiva (Ke) del modelo de infiltración de Green y Ampt y el método de la curva número $(\mathrm{CN})$, son herramientas frecuentemente utilizadas en modelos de escurrimiento y contaminación. El objetivo del presente trabajo fue analizar la relación entre Ke y CN, parámetros calibrados a partir de ensayos de infiltración realizados a campo con simulador de lluvia, determinando a su vez el efecto del cambio de escala espacial sobre dicha relación. Se trabajó con resultados de infiltración generados con un simulador de lluvia sobre suelos con aptitud agrícola y ganadera, al aplicar dos intensidades: 60 y 30 $\mathrm{mm} \cdot \mathrm{h}^{-1}$. Se calibraron los parámetros $\mathrm{CN}$ y Ke, analizando la relación entre ambos. A su vez, se trabajó con el modelo Hec Hms 4.0 para verificar si el cambio de escala espacial modificaba la relación CN-Ke obtenida previamente. En las tierras con aptitud ganadera no existió una relación significativa entre $\mathrm{CN}$ y Ke. En aquellas con aptitud agrícola se observó una relación lineal negativa y significativa entre ambos parámetros, la que dependió de la intensidad de lluvia aplicada. El aumento de escala afectó los valores de escurrimiento estimados por el modelo, respecto a los generados a campo. Sin embargo, la relación CN-Ke determinada en pequeñas parcelas, no varió significativamente al ser evaluada para una superficie mayor.
\end{abstract}

Palabras clave: Simulador de lluvia, modelo de infiltración, escurrimiento, cambio de escala

\begin{abstract}
The effective hydraulic conductivity (Ke) of the Green Ampt infiltration model and the curve number method $(\mathrm{CN})$, are tools frequently used in runoff and contamination models. The aim of the present work was to analyze the relationship between $\mathrm{Ke}$ and $\mathrm{CN}$, parameters calibrated from infiltration tests carried out in the field with a rain simulator, determining in turn the effect of the spatial scale change over this relationship. The work was carried out with infiltration results generated with a rain simulator on agricultural and livestock soils, applying two intensities: 60 and $30 \mathrm{~mm} \mathrm{~h}^{-1}$. The $\mathrm{CN}$ and Ke parameters were calibrated, analyzing the relationship between both. In turn, we worked with the Hec Hms 4.0 model to verify if the spatial scale change modified the $\mathrm{CN}-\mathrm{Ke}$ relationship previously obtained. In livestock soils there was no significant relationship between $\mathrm{CN}$ and $\mathrm{Ke}$. In those with agricultural aptitude, a significant negative linear relationship was observed, which depended on the applied rainfall intensity. It was found that the increase in scale, affected the runoff values estimated by the model, compared to those generated in the field. However, the $\mathrm{CN}-\mathrm{Ke}$ ratio obtained in small plots, did not vary significantly when was evaluated for a larger área.
\end{abstract}

Keywords: Rainfall simulator, infiltration model, runoff, scale change 


\section{INTRODUCCIÓN}

El escurrimiento y su transporte de sedimentos, nutrientes y plaguicidas, es el principal factor que contribuye a la contaminación no puntual, afectando negativamente la calidad de los cuerpos de agua superficiales (Ficklin y Zhang, 2013). La escorrentía es frecuentemente estimada a partir de la utilización del método de la curva número (CN) (USDA-NRCS, 2010), o por modelos hidrológicos que presentan alguna ecuación de infiltración, como la de Green y Ampt (Green y Ampt, 1911).

La $\mathrm{CN}$ es un modelo conceptualmente simple, basado en relaciones empíricas entre la precipitación, el uso de la tierra, el tipo de suelo y su humedad, que no considera la intensidad de la lluvia ni su duración. Contrariamente, la ecuación de Green y Ampt es un modelo de infiltración con base física, que requiere mayor detalle respecto a la dinámica temporal de la precipitación. A su vez, la $\mathrm{CN}$ asume una abstracción inicial previa al escurrimiento, mientras que con Green y Ampt se modela la escorrentía a partir de que la intensidad de la precipitación supera la tasa de infiltración (Ficklin y Zhang, 2013). La ventaja del uso de esta ecuación, es que toma en cuenta la evolución del proceso de infiltración a partir de procesos físicos que ocurren en el suelo, por lo que podría estimar mejor el impacto del cambio de uso de la tierra sobre la escorrentía (King et al., 1999).

La ecuación de infiltración de Green y Ampt es una de las más usadas en modelos hidrológicos y de erosión (Van den Putte et al., 2013; Mao et al., 2016). Su incorporación en los modelos CREAMS (Knisel, 1980), SWMM (Huber y Dickinson, 1988), SWAT (Arnold et al., 1998) y WEPP (Lane y Nearing, 1989; Laflen et al., 1997) sugiere su versatilidad (Risse et al., 1995a; Van den Putte et al., 2013). La misma presenta dos parámetros: la conductividad hidráulica efectiva $(\mathrm{Ke})$ y el potencial mátrico efectivo $(\Psi)$, respondiendo este último al contenido de agua inicial del suelo y a la tensión mátrica en el frente de humedecimiento. Se ha observado que los resultados de infiltración estimados, son sensibles a los cambios en los valores de estos parámetros, especialmente respecto a $\mathrm{Ke}$ (Nearing et al., 1990; De Roo y Jetten, 1999). Estudios previos realizados con el modelo WEPP (Van der Sweep, 1992; Risse, 1994), mostraron que es necesario un valor realista de Ke para obtener resultados de escorrentía representativos. Sin embargo, para un variado rango de suelos, los valores de Ke publicados son poco confiables, de la misma manera que está poco documentado el efecto del uso de la tierra sobre dicho parámetro (Nearing et al., 1996).

Para predecir el escurrimiento generado por 330 tormentas en 17 cuencas pequeñas, Rawls y Brakensiek (1986) usaron para estimar Ke el método desarrollado por estos mismos investigadores (Rawls y Brakensiek, 1983), logrando resultados aceptables. Sin embargo, Darder et al. (2018), al comparar los resultados de $\mathrm{Ke}$ estimados por este procedimiento con los calibrados a partir de ensayos de infiltración realizados en la región Pampeana, comprobaron que dicha metodología era poco efectiva.

La $\mathrm{CN}$ es un método ampliamente aceptado para predecir la escorrentía, pudiendo ser su valor utilizado como variable de estimación de la $\mathrm{Ke}$ (Risse et al., 1995a). En este sentido, se han realizado algunos esfuerzos para encontrar una vinculación entre ambos parámetros (Risse et al., 1995a; Zhang et al., 1995; Nearing et al., 1996). Nearing et al. (1996) desarrolló una serie de procedimientos para estimar Ke tomando en cuenta el grupo hidrológico de los suelos, su contenido de arena y el valor de CN. Estos autores demostraron utilizando esta forma de estimación de $\mathrm{Ke}$, que la predicción del volumen de escurrimiento era más realista que el solo uso de la $\mathrm{CN}$.

El objetivo del presente trabajo fue: analizar la relación entre $\mathrm{Ke}$ y $\mathrm{CN}$, parámetros calibrados a partir de ensayos de infiltración realizados a campo en pequeñas parcelas y en distintos ambientes de la cuenca alta del Arroyo Pergamino, determinando a su vez el efecto del cambio de escala espacial sobre dicho comportamiento.

\section{MATERIALES Y MÉTODOS}

\section{Área de estudio y determinaciones realizadas}

El trabajo se realizó con resultados provenientes de ensayos de infiltración efectuados a campo mediante un simulador de lluvia. Estas determinaciones se hicieron sobre suelos con aptitud agrícola y ganadera, en distintos ambientes correspondientes a la cuenca alta del Arroyo Pergamino (Pcia. de Bs. As.). El clima regional es templado, con temperaturas medias diarias que oscilan entre los 10 y $12{ }^{\circ} \mathrm{C}$ en el invierno y $23^{\circ}$ y $25^{\circ} \mathrm{C}$ en el verano. $\mathrm{La}$ precipitación media anual histórica es de $984 \mathrm{~mm}$ 
(período 1910-2015) (Estación meteorológica EEA Pergamino, INTA). En las posiciones altas del paisaje predominan Argiudoles típicos (Serie Pergamino) y sus fases con distinto grado de erosión, mientras que en los bajos los suelos forman asociaciones y complejos muy heterogéneos, que contienen proporciones variables de los suelos encontrados en las tierras altas, entremezclados con Alfisoles salinos y alcalinos (INTA, 1972).

En los ambientes ganaderos se trabajó en distintos lotes y a tres distancias respecto del curso de agua principal: $6 \mathrm{~m}, 30 \mathrm{~m}$ y $100 \mathrm{~m}$, presuponiendo la presencia de suelos con características distintas al estar dispuestos más lejos del arroyo. En los ambientes agrícolas se seleccionaron lotes bajo siembra directa ubicados en la loma y media loma, con dos sucesiones de cultivo contrastantes: predominando en algunos casos el cultivo de soja, mientras que en otros el esquema de rotación estuvo más equilibrado con gramíneas.

El simulador de lluvia utilizado posee una parcela de escurrimiento de $50 \mathrm{~cm}$ de lado (Irurtia y Mon, 1994). Se hicieron tres repeticiones por lote y por distancia al curso de agua, aplicando dos intensidades de lluvia: $60 \mathrm{~mm} \cdot \mathrm{h}^{-1}$ y $30 \mathrm{~mm} \cdot \mathrm{h}^{-1}$, en el primer caso durante una hora y en el segundo en un lapso de dos horas. En todos los casos se determinó la tasa de infiltración cada 5 minutos. No se eliminaron de la superficie del suelo los rastrojos remanentes del cultivo anterior, ni el tapiz vegetal en aquellos ambientes bajo pastura. Las simulaciones de lluvia con una intensidad de $60 \mathrm{~mm} \cdot \mathrm{h}^{-1}$ fueron realizadas en mayo de 2011, siendo en total 85 (28 en lomas, 28 en medias lomas y 29 en ambientes con aptitud ganadera). En mayo de 2013 se hicieron 74 simulaciones a $30 \mathrm{~mm} \cdot \mathrm{h}^{-1}$ (27 en lomas, 27 en medias lomas y 20 en las posiciones bajas del paisaje). En cada ensayo se tomaron muestras para la determinación de la humedad gravimétrica edáfica inicial y la porosidad del suelo superficial. Para mayor detalle de la parte metodológica ver Darder et al. (2018).

Análisis de los resultados generados con simulador de lluvia

Con los resultados de la lámina total de escurrimiento y de lluvia de cada ensayo realizado a campo, se estimó primeramente el valor de $\mathrm{S}$ (retención potencial máxima al comienzo de la tormenta), aplicando la ecuación 1 (Yoo et al., 1993):
$S=5 P+10 Q-10\left(Q^{2}+1.25 P Q\right)^{0.5}$

Siendo: $\mathrm{P}=$ lámina de lluvia $(\mathrm{mm}) ; \mathrm{Q}=$ lámina de escurrimiento $(\mathrm{mm})$.

Posteriormente, los valores de $\mathrm{S}$ fueron transformados a CN mediante la ecuación 2 (Hawkins, 1993):

$C N=\frac{25400}{(254+S)}$

El método de la CN fue desarrollado por el Servicio de Conservación de Suelos de Estados Unidos (USDA-NRCS, 2010) para predecir el escurrimiento en superficies que oscilan entre 0.0971 y 18600 ha. No obstante, en el presente trabajo se empleó una superficie menor con la finalidad de analizar fundamentalmente la relación $\mathrm{CN}-\mathrm{Ke}$ observando posteriormente su comportamiento en una mayor superficie.

A partir de los resultados de tasa de infiltración de cada ensayo de simulación de lluvia, obtenida cada cinco minutos durante una o dos horas, se calibró la conductividad hidráulica efectiva y la tensión mátrica en el frente de humedecimiento, correspondientes al modelo de infiltración de Green Ampt (Green y Ampt, 1911). Se determinó el grado de vinculación entre ambos parámetros $(\mathrm{CN}$ y $\mathrm{Ke})$ para la escala de los ensayos realizados a campo. Posteriormente se analizó el grado de ajuste entre los resultados calibrados de $\mathrm{Ke}$ y los estimados mediante la ecuación desarrollada por Nearing et al. (1996) para suelos bajo agricultura, la cual relaciona la $\mathrm{Ke}$ con $\mathrm{CN}$ según se detalla en la ecuación 3:

$K e=\left[\frac{56.82 \operatorname{Kef}^{0.286}}{1+0.051 \exp ^{(0.062 C N)}}\right]^{-2}$

Siendo: $\mathrm{Ke}=$ Conductividad hidráulica efectiva; Kef = conductividad hidráulica efectiva obtenida por tablas para una condición de barbecho; $\mathrm{CN}=$ valor de la curva número calibrada para las condiciones de cada ensayo.

\section{Utilización del modelo Hec Hms 4.0 (2010)}

Mediante el modelo hidrológico Hec Hms 4.0 (2010), se analizó si la lámina de escurrimiento 
estimada con los valores de $\mathrm{Ke}$ y $\mathrm{CN}$ calibrados con los ensayos de infiltración realizados a campo, se modificaba con la escala. Para ello, se ejecutó dicho modelo utilizando la mitad de los resultados de Ke y $\mathrm{CN}$ obtenidos según lo detallado en el punto anterior, bajo iguales condiciones iniciales pero para una superficie de 2 ha. Se contempló que los valores de estos parámetros, abarcaran el rango de resultados obtenidos a campo. Posteriormente se comparó la lámina de escurrimiento generada para las dos escalas $\left(0.25 \mathrm{~m}^{2}\right.$ y 2 ha), según los dos parámetros calibrados $\left(\begin{array}{llllll}\mathrm{Ke} & \mathrm{y} & \mathrm{CN}\end{array}\right) \mathrm{y}$ las dos intensidades de lluvia utilizadas.

También se analizó si la relación $\mathrm{Ke}-\mathrm{CN}$ obtenida con los ensayos de campo para las dos intensidades de lluvia, se mantenía con el cambio de superficie evaluada. De esta manera y con la utilización del modelo Hec Hms 4.0 (2010), se calibraron nuevamente los parámetros $\mathrm{Ke}$ y $\mathrm{CN}$ (calibración condicionada) para un área de 2 ha. Para ello se tomó en cuenta la información inicial de campo: humedad edáfica superficial inicial, hietograma de lluvia, caudal generado cada 5 minutos para la superficie de 2 ha (calculado a partir de los milímetros de escurrimiento obtenidos en la parcela del simulador de lluvia, convertidos a caudal considerando la mayor superficie), tensión mátrica en el frente de humedecimiento calibrada para los ensayos de simulación de lluvia, porosidad total del suelo y su contenido de agua bajo suelo saturado (Darder et al., 2018). Con el mismo objetivo pero sin contemplar los resultados de caudal determinados con el simulador de lluvia, se realizó una nueva calibración de Ke (calibración no condicionada), a partir de los caudales estimados con el modelo Hec Hms 4.0 para distintos valores de CN. En este caso, se consideró un rango de valores de $\mathrm{CN}$ similar al obtenido en las pequeñas parcelas, aparte de tomar en cuenta el resto de las condiciones iniciales de los ensayos realizados a campo.

El tiempo de retardo (Lag) utilizado con el modelo Hec Hms 4.0 (2010) fue calculando el tiempo de concentración por la fórmula de Sheridan (1994) (Ecuación 4), considerando posteriormente que el Lag es igual a $0.6 *$ tiempo de concentración (Chow et al., 1994).

$$
T C=2.96\left(D A^{0.54}\right)
$$

Siendo: TC (horas) = tiempo de concentración y $\mathrm{DA}=$ área de drenaje en $\mathrm{km}^{2}$.

\section{Análisis de los resultados}

El criterio utilizado para determinar los mejores resultados de $\mathrm{CN}$ y Ke calibrados, fue la utilización del coeficiente de Nash y Sutcliffe (1970) (Ecuación 5):

$R_{N}^{2}=1-\frac{\sum\left(\operatorname{Sim}_{i}-O b s_{i}\right)^{2}}{\sum\left(O b s_{i}-\text { Media }_{o b s}\right)^{2}}$

Siendo: $\mathrm{Sim}_{\mathrm{i}}$ : Valores simulados; $\mathrm{Obs}_{\mathrm{i}}$ : Valores observados; Media ${ }_{\mathrm{obs}}$ : Valor medio observado.

$\mathrm{R}^{2}{ }_{\mathrm{N}}$ puede variar entre $-\infty$ y 1 . Un valor $\mathrm{R}_{\mathrm{N}}^{2}$ igual a 1 indica una simulación perfecta (ajuste perfecto a la línea 1:1). Valores menores a cero (negativos) indican que el error del modelo es mayor que la variabilidad de la propiedad bajo estudio y por lo tanto es mejor usar el promedio de los datos que utilizar los valores simulados.

La comparación de resultados obtenidos y el grado de vinculación entre $\mathrm{Ke}$ y $\mathrm{CN}$, se hicieron a partir de análisis de varianza, correlación y regresión lineal. Para ello se utilizó el programa InfoStat versión 2017 (Di Rienzo et al., 2017).

\section{RESULTADOS Y DISCUSIÓN}

\section{Resultados generados con simulador de lluvia}

En la Tabla 1 se detallan los resultados medios de $\mathrm{CN}$ y Ke y su variabilidad, calibrados a partir de los ensayos de simulación de lluvia, junto con los de humedad gravimétrica inicial del suelo superficial.

Las tierras ganaderas presentaron una $\mathrm{Ke}$ significativamente más baja $(\mathrm{p}<0.05)$, al mismo tiempo que un valor de $\mathrm{CN}$ mayor $(\mathrm{p}<0.05)$, independientemente de la intensidad de lluvia aplicada. También se observó cierto efecto de la intensidad de lluvia sobre estos parámetros, aunque el mismo resultó significativo $(\mathrm{p}<0.05)$ únicamente para la Ke calibrada en las tierras agrícolas. La variabilidad determinada para $\mathrm{Ke}$ fue sensiblemente superior a la de CN, siendo a su vez el coeficiente de variación de este último parámetro menor en las tierras ganaderas. La humedad edáfica inicial media fue del $30-31 \%$, salvo en el caso de las tierras agrícolas bajo $30 \mathrm{~mm} \cdot \mathrm{h}^{-1}$ de lluvia simulada, en las cuales el contenido hídrico del suelo fue 
significativamente menor $(\mathrm{p}<0.05)$. Estos valores de contenido hídrico se consideraron equivalentes a los de una condición antecedente de humedad media (Condición II) (USDA-NRCS, 2010).

Tabla 1: Ke y CN medios calibrados y humedad edáfica superficial inicial.

\begin{tabular}{|l|c|c|c|c|c|c|c|c|}
\hline Intensidad lluvia & \multicolumn{4}{|c|}{$30 \mathrm{~mm} \cdot \mathrm{h}^{-1}$} & \multicolumn{4}{c|}{$60 \mathrm{~mm}^{-1}$} \\
\hline Aptitud de las tierras & \multicolumn{2}{|c|}{ Agrícolas } & \multicolumn{2}{|c|}{ Ganaderas } & \multicolumn{3}{c|}{ Agrícolas } & \multicolumn{3}{c|}{ Ganaderas } \\
\hline & Media & $\mathrm{CV}(\%)$ & Media & $\mathrm{CV}(\%)$ & Media & $\mathrm{CV}(\%)$ & Media & $\mathrm{CV}(\%)$ \\
\hline $\mathrm{Ke}\left(\mathrm{mm} \mathrm{h}^{-1}\right)$ & $10.8 \mathrm{a} \mathrm{A}$ & 71 & $1.7 \mathrm{~b} \mathrm{~A}$ & 47 & $20.2 \mathrm{a} \mathrm{B}$ & 73 & $2.2 \mathrm{~b} \mathrm{~A}$ & 101 \\
\hline $\mathrm{CN}$ & $76 \mathrm{~b} \mathrm{~A}$ & 22 & $89 \mathrm{a} \mathrm{A}$ & 17 & $78.2 \mathrm{~b} \mathrm{~A}$ & 17 & $92 \mathrm{a} \mathrm{A}$ & 5 \\
\hline $\mathrm{HE}(\%)$ & $24 \mathrm{a} \mathrm{B}$ & 23 & $30 \mathrm{~b} \mathrm{~A}$ & 40 & $30 \mathrm{a} \mathrm{A}$ & 15 & 31 a A & 39 \\
\hline
\end{tabular}

Ke: conductividad hidráulica efectiva; $\mathrm{CN}$ : curva número; HE: humedad edáfica superficial inicial; CV: coeficiente de variación. Letras minúsculas diferentes para una misma propiedad, indican diferencias estadísticas significativas entre usos distintos de la tierra para igual intensidad de lluvia $(\mathrm{p}<0.05)$. Letras mayúsculas diferentes para una misma propiedad, indican diferencias estadísticas significativas entre intensidades de lluvia distintas para igual uso de la tierra $(\mathrm{p}<0.05)$.

En las tierras con aptitud ganadera, independientemente de la intensidad de lluvia aplicada, se encontró una vinculación débil entre los resultados de estos dos parámetros calibrados. Los valores de $\mathrm{CN}$ se ubicaron mayoritariamente por encima de 85 , mientras que los de Ke oscilaron entre 0.2 y $6 \mathrm{~mm} \cdot \mathrm{h}^{-1}$. Estos resultados no difirieron de lo encontrado en la bibliografía. En este sentido, Luque et al. (1979), citados por Orsolini et al. (2000), mencionaron para suelos de ambientes bajos, inundables y con escasa infiltración, valores de $\mathrm{CN}$ entre 84 y 88 para los grupos hidrológicos de suelo $\mathrm{C}$ y D, respectivamente. Por su parte y de acuerdo a lo publicado por Rawls et al. (1983), la Ke de los suelos con textura superficial similar a los encontrados en el área evaluada (limosos y franco limosos), sería en promedio 3.4 y $6.5 \mathrm{~mm} \cdot \mathrm{h}^{-1}$. Dada la falta de vinculación entre ambos parámetros para este tipo de tierras, se prosiguió únicamente con el análisis de dicha relación para las tierras con aptitud agrícola.

En estas últimas se comprobó una relación negativa entre ambos parámetros, la cual estuvo a su vez condicionada por la intensidad de la lluvia aplicada (Figura 1), resultando mayor la Ke para un mismo valor de $\mathrm{CN}$ cuando la precipitación fue de 60 $\mathrm{mm} . \mathrm{h}^{-1}$. Sin embargo, para valores de $\mathrm{CN}$ iguales o mayores a 85 , la relación $\mathrm{Ke}-\mathrm{CN}$ fue similar para ambas intensidades de lluvia.

En este sentido y bajo $30 \mathrm{~mm} \cdot \mathrm{h}^{-1}$, para una $\mathrm{CN}$ igual o mayor a 85 , los resultados de Ke estuvieron dentro del rango $0.2-8 \mathrm{~mm} \cdot \mathrm{h}^{-1}$, mientras que para $60 \mathrm{~mm} \cdot \mathrm{h}^{-1}$ se registraron valores de $\mathrm{Ke}$ en el rango de 0.7-9 $\mathrm{mm} \cdot \mathrm{h}^{-1}$. Este comportamiento coincide con lo hallado para las tierras ganaderas: una Ke reducida para un acotado intervalo de elevados valores de $\mathrm{CN}$.

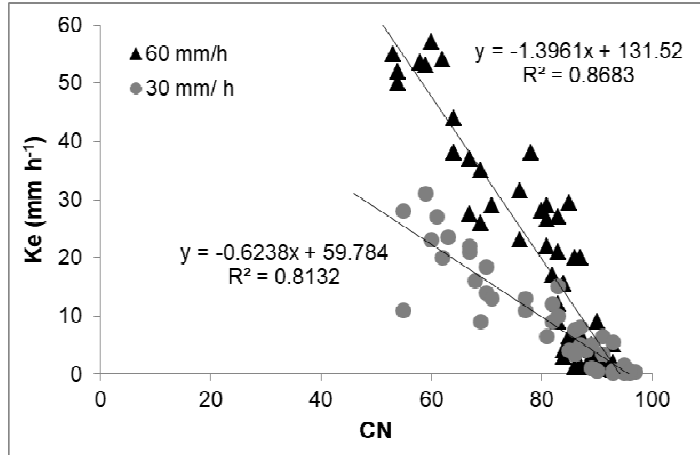

Figura 1. Relación entre CN y Ke para las tierras con aptitud agrícola, considerando dos intensidades de lluvia.

A su vez, la diferente sucesión de cultivos y la posición en el paisaje, no modificaron la tendencia general encontrada para la relación Ke-CN (Figuras 2 y 3 ) en las tierras agrícolas. Por su parte, los resultados de estos dos parámetros no estuvieron condicionados por el diferente uso de la tierra o por la posición en el paisaje de los suelos, dado que sus valores abarcaron un rango similar, independientemente si los suelos se ubicaban en la loma o media loma, o si se encontraban bajo el monocultivo de soja o con una sucesión de cultivos más equilibrada con gramíneas.

La amplitud de resultados de la Ke y $\mathrm{CN}$ calibradas para las tierras bajo agricultura, excedió lo publicado en la bibliografía. Luque et al. (1979), citados por Orsolini et al. (2000), mencionan para suelos pertenecientes al grupo hidrológico $\mathrm{C}$, con cultivos de verano y bajo una condición antecedente de humedad II, valores de $\mathrm{CN}$ entre 72 y 82 , dependiendo del grado de la pendiente del terreno. Por su parte y de acuerdo con Rawls et al. (1983), la 
Ke de estas tierras sería de igual magnitud a la mencionada para las ganaderas, dada la similar textura superficial de ambos tipos de suelo. De esta manera, los valores de $\mathrm{Ke}$ y $\mathrm{CN}$ citados por estos autores, representarían entre el 10 y $23 \%$ de los calibrados a partir de las mediciones de infiltración realizadas a campo. Esta mayor variabilidad no pudo ser explicada a partir del diferente contenido hídrico inicial de los suelos, ya que el análisis de correlación mostró que el intervalo de humedad edáfica registrado, no incidió significativamente sobre Ke y CN. Solo bajo $30 \mathrm{~mm} . \mathrm{h}^{-1}$ de lluvia simulada, se comprobó una débil relación entre la humedad del suelo y $\mathrm{Ke}\left(\mathrm{R}^{2}\right.$ : 0.17).

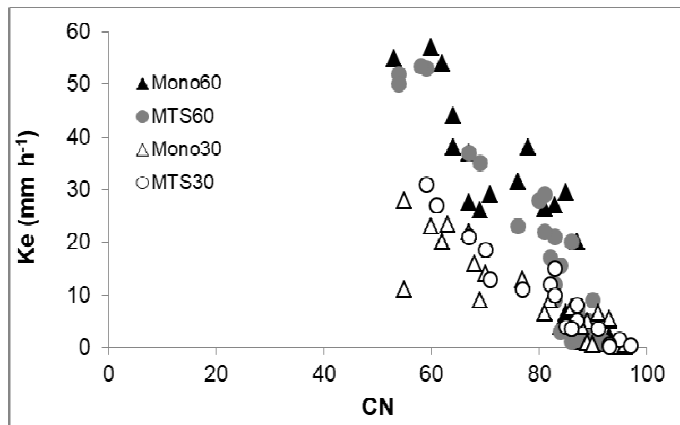

Figura 2. Relación entre la CN y Ke para las tierras agrícolas, de acuerdo a la diferente sucesión de cultivos.

Mono60 y Mono30: monocultivo de soja con $60 \mathrm{~mm} \cdot \mathrm{h}^{-1}$ y 30 $\mathrm{mm} \cdot \mathrm{h}^{-1}$ de lluvia aplicada, respectivamente. MTS60 y MTS30 rotación equilibrada de gramíneas y leguminosas con $60 \mathrm{~mm} . \mathrm{h}^{-1} \mathrm{y}$ $30 \mathrm{~mm} \cdot \mathrm{h}^{-1}$ de lluvia aplicada, respectivamente.

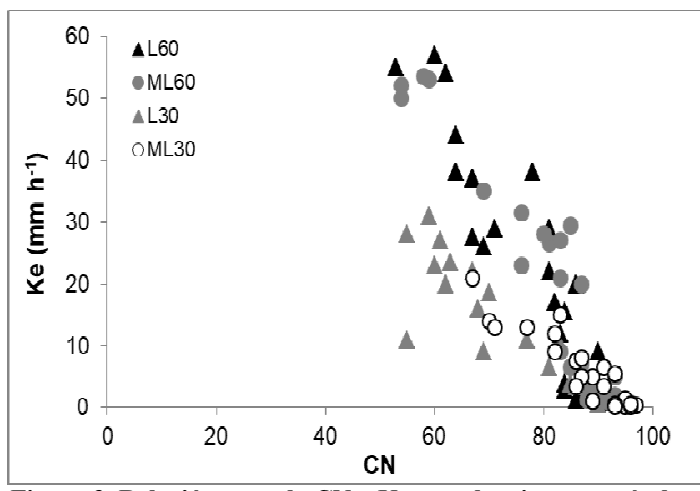

Figura 3. Relación entre la $\mathrm{CN}$ y Ke para las tierras agrícolas, de acuerdo a la distinta posición en el paisaje.

L60 y L30: Posición loma con $60 \mathrm{~mm} \cdot \mathrm{h}^{-1}$ y $30 \mathrm{~mm} \cdot \mathrm{h}^{-1}$ de lluvia aplicada, respectivamente. ML60 y ML30: Posición media loma con $60 \mathrm{~mm} \cdot \mathrm{h}^{-1}$ y $30 \mathrm{~mm} \cdot \mathrm{h}^{-1}$ de lluvia aplicada, respectivamente.

Distintos trabajos muestran que el tamaño del soporte influye en la variabilidad de los resultados de conductividad hidráulica saturada (Baveye y
Spósito, 1984; Mallants et al., 1997). Por lo tanto, la amplitud de valores observada en $\mathrm{Ke}$ y $\mathrm{CN}$, obedecería en parte a que el reducido tamaño de la parcela empleada en las mediciones de campo, habría capturado una mayor diversidad de situaciones, las que habrían quedado diluidas si se hubiese trabajado sobre una superficie mayor.

Los sistemas de producción agrícola en la región Pampeana se encuentran bastante estandarizados, reduciendo la heterogeneidad de las condiciones edáficas. Sin embargo, en el presente trabajo hubo factores que no fueron identificados, que favorecieron el aumento en la variabilidad de los parámetros que condicionan la relación lluviaescurrimiento ( $\mathrm{CN}$ y $\mathrm{Ke})$. En este sentido, algunos investigadores (Risse et al., 1995b; Zhang et al., 1995) desarrollaron algunos algoritmos para predecir los cambios sufridos en la Ke, a partir de modificaciones ocurridas en la estructura del suelo, la rugosidad superficial, la intensidad del encostramiento superficial y el grado y tipo de la cobertura vegetal.

Al aplicar la fórmula desarrollada por Nearing et al. (1966) para suelos agrícolas, se comprobó una subestimación de la $\mathrm{Ke}$ respecto a los valores calibrados de este parámetro (Figura 4).

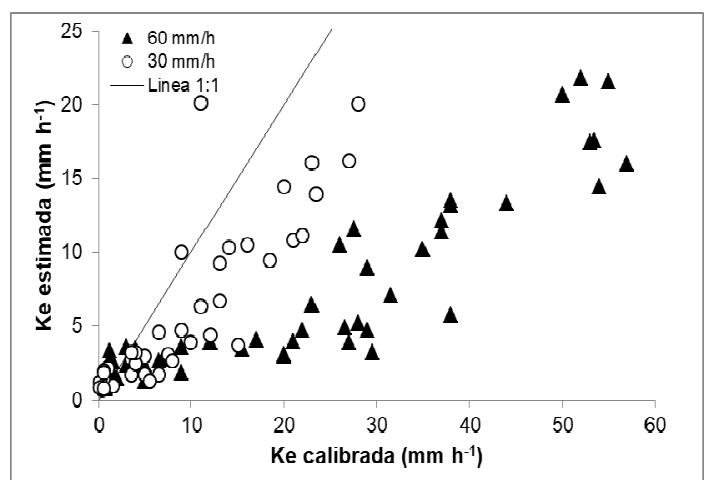

Figura 4. Relación entre la Ke estimada de acuerdo a Nearing et al. (1966) y la Ke calibrada para las tierras agrícolas.

Este comportamiento se observó con mayor intensidad en los ensayos con $60 \mathrm{~mm} \cdot \mathrm{h}^{-1}$ de lluvia simulada. En este sentido, desde valores muy bajos de la Ke calibrada y hasta los $30 \mathrm{~mm} \cdot \mathrm{h}^{-1}$ de este parámetro, se observó un ligero incremento de la Ke estimada por fórmula. Sin embargo, a partir de dicho valor se pudo comprobar una tendencia en los resultados de la relación Ke estimada-Ke calibrada, bastante similar a la línea de perfecto ajuste (1:1). 
Por su parte, con una intensidad de lluvia de 30 $\mathrm{mm} . \mathrm{h}^{-1}$, si bien esta relación también se ubicó por debajo de la mencionada línea, la distancia respecto a la misma fue menor.

\section{Utilización del modelo Hec Hms 4.0 (2010)}

La relación entre las láminas de escurrimiento observadas a campo y estimadas por el modelo Hec Hms 4.0 para una superficie de 2 ha, dependió del parámetro empleado $(\mathrm{CN}$ o $\mathrm{Ke})$ y de la intensidad de lluvia aplicada (Figuras 5 y 6 ).

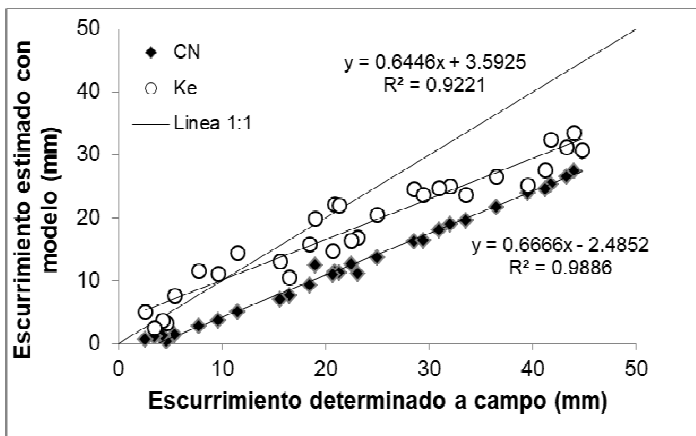

Figura 5. Relación entre las láminas de escurrimiento generadas para ambas escalas bajo una intensidad de lluvia de $60 \mathrm{~mm} \cdot \mathrm{h}^{-1}$.

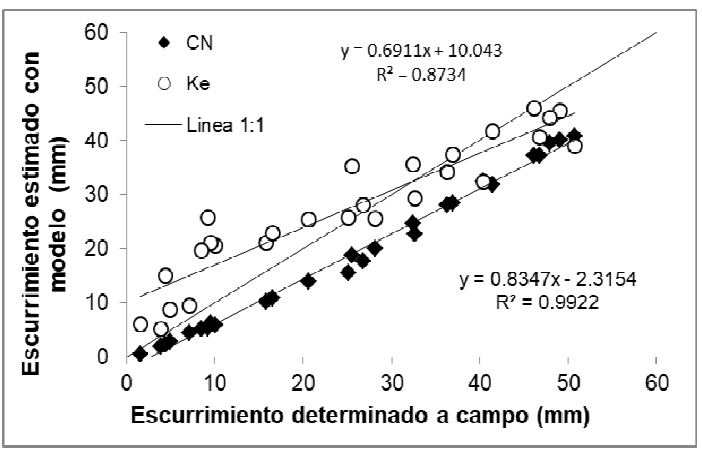

Figura 6. Relación entre las láminas de escurrimiento generadas para ambas escalas bajo una intensidad de lluvia de $30 \mathrm{~mm} \cdot \mathrm{h}^{-1}$.

Con una precipitación de $60 \mathrm{~mm} \cdot \mathrm{h}^{-1}$ (Figura 5) y para iguales valores de $\mathrm{CN}$, el escurrimiento estimado por $\mathrm{Hec} \mathrm{Hms} 4.0$ fue siempre menor al registrado en las parcelas del simulador de lluvia.

A su vez, este comportamiento se acentuó en la medida que el escurrimiento fue mayor. Por su parte, los milímetros escurridos estimados con dicho modelo al utilizar Ke, fueron bastante similares para ambas escalas hasta una lámina de $12 \mathrm{~mm}$, a partir de la cual las diferencias se incrementaron. Al usar
$\mathrm{CN}$, los milímetros de escurrimiento estimados estuvieron entre el 32 y $1577 \%$ por debajo de los registrados a campo (promedio: $51 \%$ ), mientras que utilizando $\mathrm{Ke}$ los valores extremos estuvieron por arriba $(90 \%)$ y por debajo (37\%) de lo observado en las parcelas con menor superficie (promedio: 11\%).

El coeficiente de Nash y Sutcliffe (1970), obtenido al contrastar los valores de escurrimiento para ambas escalas, fue 0.29 y 0.71 al utilizar $\mathrm{CN}$ y $\mathrm{Ke}$, respectivamente.

Con una precipitación de $30 \mathrm{~mm} \cdot \mathrm{h}^{-1}$ (Figura 6), los resultados estimados de escurrimiento a partir de $\mathrm{CN}$ presentaron un comportamiento similar al observado con una intensidad de lluvia mayor, aunque en este caso los porcentajes en que se redujo la lámina escurrida estimada con el aumento de escala oscilaron entre el 17 y $75 \%$ (promedio: 33\%). Por su parte, con $\mathrm{Ke}$ las mayores diferencias de estimación estuvieron entre $-23 \%$ y $+276 \%$ (promedio: 46\%). Para esta intensidad de lluvia, los resultados del coeficiente de Nash y Sutcliffe (1970) obtenidos al comparar el escurrimiento entre escalas, fueron muy similares al utilizar $\mathrm{Ke}$ o $\mathrm{CN}$ (0.82 y 0.80 , respectivamente).

De acuerdo con estos resultados, los valores de Ke y $\mathrm{CN}$ calibrados serían diferentes de acuerdo a la superficie considerada. En este sentido, Castiglioni (2016) determinó una reducción en la Ke calibrada del 60 al $100 \%$, al pasar de una superficie de $0.25 \mathrm{~m}^{2}$ a otra de $2100 \mathrm{~m}^{2}$. A su vez, este comportamiento dependería también de la intensidad de lluvia y de la magnitud del escurrimiento generado (Figuras 5 y 6). Considerando los coeficientes de Nash y Sutcliffe (1970) obtenidos, la utilización de la Ke calibrada a una determinada escala, arrojaría menos incertidumbre al estimar el escurrimiento a una escala mayor, en comparación al uso de la $\mathrm{CN}$. No obstante, para poder llegar a conclusiones más generales, sería necesario aportar más resultados que abarquen una mayor diversidad de situaciones.

Estas diferencias no fueron suficientes para modificar la relación $\mathrm{CN}-\mathrm{Ke}$ previamente observada (Figuras 1, 7 y 8).

Estos parámetros, calibrados mediante el modelo Hec Hms 4.0 con los datos de lámina de lluvia y de escurrimiento obtenidos con el simulador de lluvia y transformados a caudal para una superficie de 2 ha, mantuvieron dicha relación (Figura 1) independientemente de la intensidad de lluvia 
aplicada (Figuras 7 y 8). Lo mismo sucedió al calibrar la Ke mediante el mismo modelo, a partir de los caudales estimados para una superficie de 2 ha con distintos valores de $\mathrm{CN}$, no estando estos directamente vinculados con los resultados obtenidos a campo (Figuras 7 y 8).

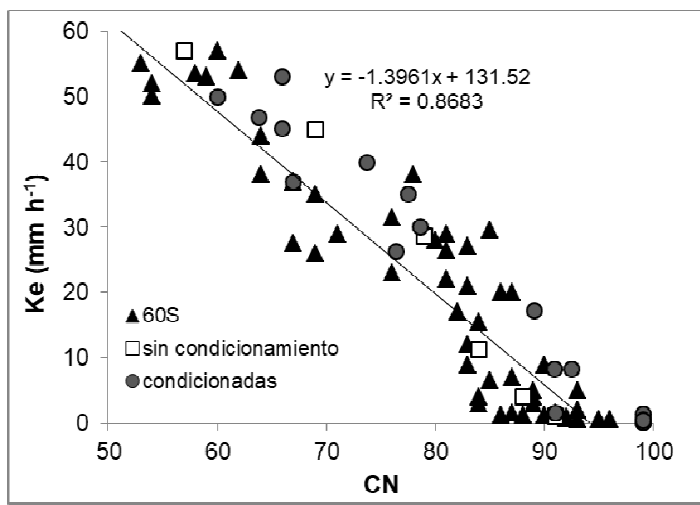

Figura 7. Relación entre CN y Ke calibradas a dos escalas espaciales para una intensidad de $60 \mathrm{~mm} . \mathrm{h}^{-1}$.

60S: Relación entre $\mathrm{CN}$ y Ke, generada a partir de los ensayos de simulación de lluvia a campo; sin condicionamiento: Relación entre $\mathrm{CN}$ y $\mathrm{Ke}$, calibradas con el modelo Hec Hms 4.0 para una superficie de 2 ha, sin estar los resultados condicionados por los caudales observados a campo; condicionadas: Relación entre $\mathrm{CN}$ y Ke, calibradas con el modelo Hec Hms 4.0 para una superficie de 2 ha, estando los resultados condicionados por los caudales observados a campo.

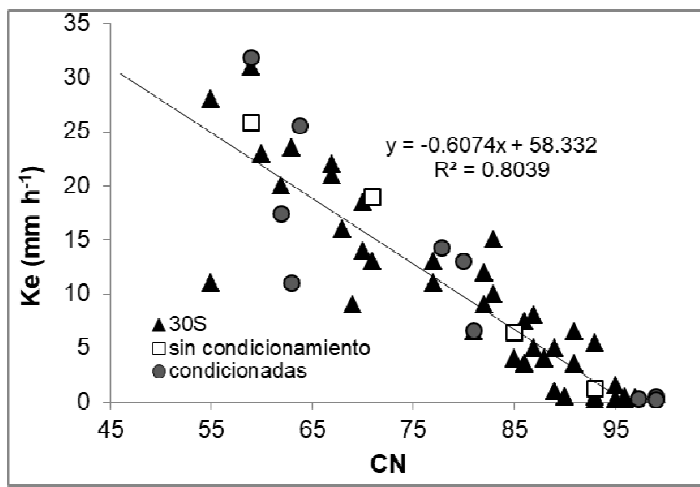

Figura 8. Relación entre CN y Ke calibradas a dos escalas espaciales para una intensidad de $30 \mathrm{~mm} \cdot \mathrm{h}^{-1}$.

30S: Relación entre CN y Ke, generada a partir de los ensayos de simulación de lluvia a campo; sin condicionamiento: Relación entre $\mathrm{CN}$ y $\mathrm{Ke}$, calibradas con el modelo $\mathrm{Hec} \mathrm{Hms} 4.0$ para una superficie de 2 ha, sin estar los resultados condicionados por los caudales observados a campo; condicionadas: Relación entre $\mathrm{CN}$ y Ke, calibradas con el modelo Hec Hms 4.0 para una superficie de 2 ha, estando los resultados condicionados por los caudales observados a campo.

A su vez, el grado de dispersión obtenido de Ke y $\mathrm{CN}$ no fue mayor que el determinado con las parcelas más pequeñas. Estos resultados muestran la alta vinculación que existe entre la $\mathrm{CN}$ y $\mathrm{Ke}$ independientemente de la escala, pudiendo por lo tanto la $\mathrm{CN}$ ser utilizada como una variable para predecir Ke.

\section{CONCLUSIONES}

Con el objetivo de analizar la relación entre $\mathrm{CN}$ y $\mathrm{Ke}$, parámetros calibrados a partir de ensayos de infiltración realizados a campo sobre suelos representativos de la Pampa Ondulada, se pudo comprobar en las tierras con aptitud ganadera, que no existió una relación significativa entre ambas variables. Sin embargo, en aquellos suelos con aptitud agrícola, se observó una correlación lineal negativa y significativa entre $\mathrm{CN}$ y $\mathrm{Ke}$, la que fue afectada por la intensidad de lluvia aplicada. No obstante, para resultados de $\mathrm{CN}$ superiores a 85 , las diferencias en dicha relación entre intensidades de lluvia se diluyeron. Los valores calibrados de estos parámetros, presentaron en las tierras agrícolas una amplitud mayor que la publicada en la bibliografía. La ecuación desarrollada por Nearing et al. (1996) subestimó los valores de $\mathrm{Ke}$, lo cual fue más evidente bajo una intensidad de lluvia de $60 \mathrm{~mm} \cdot \mathrm{h}^{-1}$. La relación $\mathrm{CN}-\mathrm{Ke}$ obtenida en pequeñas parcelas, se comportó de manera similar al ser estimada para una superficie mayor, no obstante lo cual los resultados obtenidos en el presente trabajo deberían ser convalidados con un mayor número de ensayos y que abarquen una mayor diversidad de situaciones.

\section{REFERENCIAS BIBLIOGRÁFICAS}

Arnold, J. G., Srinivasan, R., Muttiah, R. S., Williams, J. R. (1998). Large area hydrologic modeling and assessment Part 1: Model development. J.Am. Water Resour. As., 34(1), 73-89.

Baveye, P. y Sposito, G. (1984). The operational significance of the continuum hypothesis in thetheory of wáter movement through soils and aquifers. Water Resources Research, 20 (5), 521-530.

Castiglioni, M. (2016). Propiedades físicas y conductividad hidráulica efectiva en el transcurso de una rotación trigo/soja. Cuadernos del Curiham, 22, 15-25.

Chow, V. T., Maidment, D. R. y Mays, L. W. (1994). Hidrología aplicada. Suárez, M. E. (Ed). McGraw Hill, Interamericana S.A., 584 págs.

Darder, M. L., Castiglioni, M., Andriulo, A. y Sasal, M. C. (2018). Calibración de parámetros de un modelo de infiltración en la cuenca alta del Arroyo 
Pergamino. Ciencia del Suelo. En revisión.

De Roo, A. P. J. y Jetten, V. G. (1999). Calibrating and validating the LISEM model for two data sets from the Netherlands and South Africa. Catena, 37(3-4), 477-493.

Di Rienzo, J. A., Casanoves, F., Balzarini, M. G., Gonzalez, L., Tablada, M. y Robledo, C. W. (2017). InfoStat versión 2017. Grupo InfoStat, FCA, Universidad Nacional de Córdoba, Argentina. URL http://www.infostat.com.ar

Ficklin, D. L. y Zhang, M. (2013). A comparison of the curve number and Green-Ampt models in an agricultural watershed. Trans. ASABE, 56(1), 61-69.

Green, W. H. y Ampt, G. (1911). Studies in soil physics. I: The flow of air and water through soils. J. Agr. Sci., 4, 1-24.

Hawkins, R. (1993). Asymptotic determination of runoff curve numbers from data. J. Irrig. Drainage Eng., 19(2), 334-345.

Hec-Hms 4.0. (2010). Hydologic Modeling System. U.S. Army Corps of Engineers. Institute for Water Resource. Hydrologic Engineering Center. Davis C.A.

Huber, W. C. y Dickinson, R. E. (1988). Storm Water Management Model, Version 4: User's Manual. Environmental Research Laboratory. U.S. Environmental Protection Agency, Athens, Georgia.

INTA. (1972). Cartas de Suelo de la República Argentina. Hoja 3360-32 Pergamino, 106p.

Irurtia, C.B. y Mon, R. 1994. Microsimulador de lluvia para determinar infiltración a campo. Instituto de Suelos. CIRN INTA Castelar. Publicación Técnica 176.18 pp.

King, K. W., Arnold, J. G. y Bingner, R. L. (1999). Comparison of Green-Ampt and curve number methods on Goodwin Creek watershed using SWAT. Trans. ASAE., 42(4), 919-925.

Knisel, W. (1980). CREAMS. A Field-Scale Model for Chemicals, Runoff and Erosion from Agricultural Management Systems. USDA Conservation Research Report, No.26, 640 pp.

Laflen, J. M., Elliot, W. J., Flanagan, D. C., Meyer, C. R. y Nearing, M. A. (1997). WEPP predicting water erosion using a process-based model. J. Soil Water. Conserv., 52 (2), 96-102.

Lane, L. J. y Nearing, M. (Editors). (1989). USDA Water Erosion Prediction Project: Hillslope Profile Model Documentation. NSERL Report No. 2, National Soil Erosion Research Laboratory, West Lafayette, Indiana.

Luque, J. A., Paoloni, J. D. y Bonorino, G. A. (1979). Estudio geológico e hidrogeológico de la cuenca del Río Sauce Grande. Publicación interna del Departamento de Ciencias Agrarias y de Ciencias
Naturales de la Universidad Nacional del Sur, Serie Hidrología. Bahía Blanca, Argentina, 3: 64 p.

Mallants, D., Binayak, P., Mohanty, B. P., Vervoort, A. y Feyen, J. (1997). Spatial analysis of saturated hydraulic conductivity in a soil with macropores. Soil Technol., 10, 115-131.

Mao, L., Li, Y., Hao, W., Zhou, X., Xu, C. y Lei, T. (2016). A new method to estimate soil water infiltration based on a modified Green-Ampt model. Soil Till. Res., 161, 31-37.

Nash, J. E. y Sutcliffe, J. V. (1970). River Flow Forecasting Through Conceptual Models, Part 1, A Discussion of Principles. J. Hydrol., 10, 282-290.

Nearing, M. A., Deer-Ascough, L. y Laflen, J. M. (1990). Sensitivity analysis of the WEPP hillslope profile erosion model. Trans. ASAE, 33(3), 839-849.

Nearing, M. A., Liu, B. Y., Risse, L. M. y Zhang, X. (1996). Curve numbers and Green-Ampt effective hydraulic conductivities. Water Resour. Bull., 32(1), 125-136.

Orsolini, H. E., Zimmermann, E. D. y Basile, P. A. (2000). Hidrología, procesos y métodos. UNR (Ed). Rosario. 319 pp.

Rawls, W. J. y Brakensiek, D. L. (1983). A procedure to predict Green and Ampt infiltration parameters. In Proc. ASAE Conf. On Advances in Infiltration, ASAE, St. Joseph, Michigan, pp. 102-112.

Rawls, W. J., Brakensiek, D. L. y Miller, N. (1983). Green Ampt infiltration parameters from soils data. J Hidraul. Div., Am. Soc. Civ. Eng., 109(1), 62-70.

Rawls, W. J. y Brakensiek, D. L. (1986). Comparison Between Green-Ampt and Curve Number Runoff Predictions. Trans. ASAE, 29(6), 1597-1599.

Risse, L. M. (1994). Validation of WEPP Using Natural Runoff Plot Data. Unpublished Ph.D. Dissertation, National Soil Erosion Research Laboratory, Purdue University, West Lafayette, Indiana, $230 \mathrm{p}$.

Risse, L. M., Liu, B. Y. y Nearing, M. A. (1995a). Using curve numbers to determine baseline values of Green-Ampt effective hydraulic conductivities. Water Resour. Bull., 31(1), 147-158.

Risse, L. M., Nearing, M. A. y Zhang, X. C. (1995b). Variability in Geen-Ampt effective hydraulic conductivity under fallow conditions. $J$. Hydrol, 169, 1-24.

Sheridan, J. M. (1994). Hydrograph time parameters for flatland watersheds. Trans. ASAE 37, 103-113.

USDA-NRCS. (2010). National Engineering Handbook. Section 4: Hydrology. National Soil Conservation Service, USDA, Washington, DC. 
Van den Putte, A, Govers, G., Leys, A., Langhans, C., Clymans, W. y Diels, J. (2013). Estimating the parameters of the Green-Ampt infiltration equation from rainfall simulation data: Why simpler is better. J. Hydrol, 476, 332-344.

Van der Zweep, R. A. (1992). Evaluation of the Water Erosion Prediction Project's Hydrologic Component on a Semi Arid Rangeland Watershed. Unpublished M.S. Thesis, University of Arizona, Tucson, Arizona.

Yoo, K. H., Yoon, K. S. y Soileau, J. M. (1993). Runoff curve numbers determined by three methods under conventional and conservation tillage. Trans. ASAE, 36(1), 57-63.

Zhang, X. C.; Nearing, M. A. y Risse, L. M. (1995). Estimation of Green-Ampt conductivity parameters: Part I. Row Crops. Trans. ASAE, 38(4), 1069-1077.

\section{REGISTRO BIBLIOGRÁFICO}

Darder, M. L., Castiglioni, M., Andriulo, A. y Nasal, M. C. (2018). Análisis de la relación entre la conductividad hidráulica efectiva y la curva número bajo dos intensidades de lluvia. Cuadernos del CURIHAM. 24, 110. DOI: $10.35305 /$ curiham.v24i0.111

Tipo de Publicación: ARTICULO.

Trabajo recibido el 11/06/2018 y aprobado para su publicación el 30/11/2018. 